\title{
In vitro ANTIMICROBIAL RESISTANCE OF Pseudomonas aeruginosa ISOLATED FROM CANINE OTITIS EXTERNA IN RIO DE JANEIRO, BRAZIL
}

\author{
B. Penna ${ }^{1}$, S. Thomé ${ }^{1}$, R. Martins ${ }^{1}$, G. Martins ${ }^{1}$, W. Lilenbaum ${ }^{1}$ \\ ${ }^{1}$ Laboratório de Bacteriologia Veterinária, Universidade Federal Fluminense, Niterói, RJ, Brasil
}

Submitted: October 11, 2010; Returned to authors for corrections: March 14, 2011; Approved: May 30, 2011.

\begin{abstract}
Isolates of Pseudomonas aeruginosa (167) were obtained from 528 samples of canine otitis externa, identified by biochemical reactions and tested for susceptibility to 10 antimicrobials. The most effective drug was ciprofloxacin. The study reports alarming resistance among $P$. aeruginosa isolated from canine otitis externa samples in Rio de Janeiro, Brazil.
\end{abstract}

Key words: Pseudomonas aeruginosa, resistance, antimicrobial agents, dog, otitis externa

Canine otitis externa is one of the most frequent canine infections, and is estimated to affect 5 to $20 \%$ of the dogs. It commonly occurs as a secondary complication of primary factors that initiate inflammation within ears, such as atopic dermatitis, cutaneous adverse reactions to food, foreign bodies, ectoparasites, metabolic diseases, keratinization abnormalities, and autoimmune diseases (5).

The most common pathogens in the etiology of otitis include members of the Staphylococcus genus $(8,9)$ and Gramnegative rods. Despite Gram-negative microorganisms are not routinely cultured from the normal ear canal, Proteus mirabilis, Klebsiella pneumoniae, Escherichia coli and Pseudomonas aeruginosa have been described and cannot be neglected as agents of canine otitis externa (5).

Pseudomonas aeruginosa is a Gram-negative rod which is nonspore forming and aerobe. In dogs, P. aeruginosa have already been reported on otitis media/external infections $(4,6)$.
The bacterium can be resistant to all classes of antimicrobial agents making it especially difficult to successfully treat patients with compromised immune defenses (8). Due to the presence of several drug efflux systems and porins, $P$. aeruginosa is intrinsically resistant to a wide range of antimicrobials including benzylpenicillins, aminobenzylpenicillins, carboxypenicillins, $1^{\text {st }}$ and $2^{\text {nd }}$ generation cephalosporins, chloramphenicol and tetracyclines (6).

The purpose of the present study was to determine the antimicrobial resistance of Pseudomonas aeruginosa isolates obtained from dogs with otitis externa in Rio de Janeiro, Brazil.

Samples were obtained from 528 adult ( $\geq$ two years) dogs unmedicated for at least 15 days diagnosed with otitis externa (local pain, pruritus, erythema, ear discharge or desquamation). The samples were collected over a 2-year period (20072009). Cotton swabs were used to collect samples, inoculated

*Corresponding Author. Mailing address: Veterinary Bacteriology Laboratory, Rua Prof. Hernani Mello, 101 sala 309, Niterói - RJ, Brazil, CEP 24210-130.; Tel: +55.21.2629-2435 Fax: +55.21.2629-2432.; E-mail: mipwalt@vm.uff.br 
into Brain Heart Infusion broth (Difco - New Jersey, USA) and incubated at $37^{\circ} \mathrm{C}$. Only one sample from each dog was studied, even if both ears presented with clinical signs. If bacterial growth occurred after $24 \mathrm{~h}$ of incubation, smears were made, Gramstained, and examined microscopically. Samples with morphology of Gram-negative rods were transferred to MacConkey's agar and Pseudomonas agar (Merck - New Jersey, USA). Isolates in pure culture were identified on the basis of colony morphology, Gram staining, pigment production (pyocyanine and pyoverdin), oxidase test, motility, aerobic fermentation of glucose, lactose, maltose and mannitol, arginine and lysine utilization, nitrate reduction, production of urease and DNAse, ONPG (orthonitrophenyl-betaD-galactopyranoside) test, acetamide and esculin hidrolysis, and susceptibility to polimyxin, according to Koneman et al. (7).

Antimicrobial susceptibility to a panel of 10 antimicrobial agents most commonly used in our community and according to
CLSI recommendation was determined (2,3) (Table 1). Aminoglycosides were represented by amikacin (AMI - 30 $\mu \mathrm{g}$ )

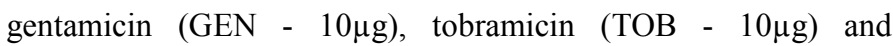
neomycin (NEO - 30 $\mu \mathrm{g}$ ), while fluoroquinolones were represented

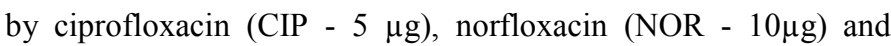

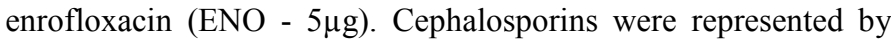

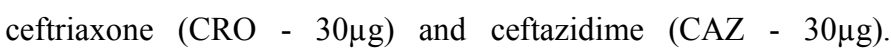

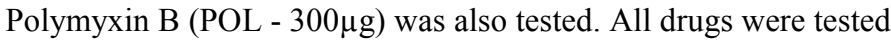
to all isolates with exception of amikacin, ceftriaxone and ceftazidime that were tested to 125 samples. Others drugs with anti - Pseudomonas activity such as Carbapenems (Imipenem and Meropenem), Cephems, Monobactams and Lipopeptides were not tested in this study since they are not commonly prescribed in the veterinary practice. After measuring the antimicrobial zone diameters, the isolates were categorized as sensitive or resistant to the drug.

Table 1. Antimicrobial resistance of 167 Pseudomonas aeruginosa isolates obtained from 528 unmedicated dogs with otitis externa from Rio de Janeiro, Brazil

\begin{tabular}{lcc}
\hline Antimicrobial & \multicolumn{2}{c}{ Resistance } \\
\cline { 2 - 3 } & $\mathbf{N}$ & $\mathbf{\%}$ \\
\hline CIP & 23 & 13.8 \\
ENO & 106 & 63.6 \\
NOR & 31 & 18.4 \\
GEN & 119 & 71.4 \\
NEO & 158 & 94.7 \\
TOB & 109 & 65.4 \\
AMI* & 85 & 70.4 \\
POL & 91 & 54.4 \\
CRO* & 76 & 60.8 \\
CAZ* & 66 & 52.8 \\
\hline * tested for 125 of the P. aeruginosa isolates. & \\
CIP - Ciprofloxacin; ENO - Enrofloxacin; NOR - Norfloxacin; GEN - Gentamicin; NEO - \\
Neomycin; TOB - Tobramycin; AMI - Amikacin; POL - Polymyxin B; CRO - Ceftriaxone; CAZ \\
- Ceftazidime
\end{tabular}

Hundred and sixty-seven out of the 528 studied samples yielded Pseudomonas aeruginosa in pure culture, representing $31.6 \%$ of the total isolates. These isolates were all resistant to multiple antimicrobial classes, including intrinsic as well as acquired resistance to newer synthetic antimicrobial agents that are commonly used in canine practice.

Highly variable susceptibility profiles were observed among these isolates, what has been extensively discussed (11, 12). For example, some isolates were resistant to the aminoglycosides (amikacin, gentamicin, and tobramycin) while susceptible to the fluoroquinolones and vice versa.

With regards to cephalosporins, significant rates of resistance were presented, with $60.8 \%$ resistant isolates to ceftriaxone and $52.8 \%$ to ceftazidime. These results agrees 
with other studies which also observed high rates of resistance to cephaloporins ranging from $97 \%$ to $100 \%$ (11).

Resistance to aminoglycosides was frequently observed; neomycin and gentamicin were the less effective drugs of this class, with $94.7 \%$ and $71.4 \%$ of the isolates being resistant, respectively. Additionally, tobramycin did not present good efficiency rates, since $65.4 \%$ of the isolates were resistant to this drug. These findings clearly contrast with findings of a recent study conducted in Canada (11) that reported $P$. aeruginosa isolates coming from canine otitis externa to be highly susceptible to those drugs (11).

In relation to the class of the fluoroquinolones, ciprofloxacin and norfloxacin demonstrated very similar rates of resistance, with $13.8 \%$ and $18.4 \%$ of resistant strains, respectively, while ciprofloxacin was the most effective drug of this study. The high susceptibility to the fluoroquinolones was expected, since other studies also highlight the efficiency of drugs from this class in the treatment of $P$. aeruginosa canine infections (13). Ciprofloxacin have been reported (11) to be the best drug among the fluoroquinolones, with only $16 \%$ of isolates presenting resistance to this drug.

What was unexpected was the high rate of resistance to enrofloxacin, since $63.6 \%$ of the isolates recovered in this study were resistant to this drug, what contrasts to other reports (11) of only $31 \%$ of resistant isolates to enrofloxacin. This phenomenon of a high resistance to enrofloxacin not being accompanied by other fluoroquinolones has already been reported in studies regarding canine hosts (13).

In conclusion, the antimicrobial resistance of $P$. aeruginosa isolates taken from cases of canine otitis externa demonstrates an alarming scenario in Rio de Janeiro, Brazil. Those data would also argue for the early consideration of culture and sensitivity testing in the management of cases of otitis externa that are not responding to empiric therapy.

\section{ACKNOWLEDGEMENTS}

This study was founded by CNPq and CAPES. WL is a research fellow of CNPq.

\section{REFERENCES}

1. Barrasa, J.L.; Gomez, P.L.; Lama, Z.G.; Junco, M.T.J. (2000). Antibacterial susceptibility patterns of Pseudomonas strains isolated from chronic canine otitis externa. J. Vet. Med. B. 47, 191-196.

2. CLSI. (2008). Performance Standards for Antimicrobial Disk and Dilution Susceptibility Test for Bacteria Isolated From Animals; Approved Standard Document M31-A3 - 3rd ed., volume 28, No 8.

3. CLSI. (2010). Performance Standards for Antimicrobial Susceptibility Test ing; Twentieth Information Supplement. M100-S20. 30 (1).

4. Galles, A.C.; Jones, R.N.; Turnidge, J.; Rennie, R.; Ramphal. R. (2001) Characterization of Pseudomonas aeruginosa isolates: occurrence rates, antimicrobial susceptibility patterns, and molecular typing in the global SENTRY antimicrobial surveillance program, 1997-1999. Clin. Infect. Dis. 32, 146-155.

5. Graham-Mize, C.A.; Rosser Jr, E.J. (2004). Comparison of microbial isolates and susceptibility patterns from the external ear canal of dogs with otitis externa. J. Am. Anim. Hosp. Assoc. 40, 102-108.

6. Hillier, A.; Alcorn, J.R.; Cole, L.K.; Kowalski, J.J. (2006) Pyoderma caused by Pseudomonas aeruginosa infection in dogs: 20 cases. Vet. Dermatol. 17, 432-439.

7. Koneman, E.W.; Allen, D.S.; Janda, W.M.; Schreckenberger, P.C.; Winn Jr, W.C. (2006). Koneman's Color Atlas and Textbook of diagnostic Microbiology $6^{\mathrm{a}}$ Ed, Lippincott - Raven publishers, Philadelphia, USA, p. 303-391.

8. Lyskova, P.; Vydrzalova, M.; Mazurova, J. (2007). Identification and antimicrobial susceptibility of bacteria and yeasts isolated from healthy dogs and dogs with otitis externa. J. Am. Vet. Med. Assoc. 54, 559-563.

9. Penna, B.; Varges, R.; Medeiros, L.; Martins, G.M.; Martins, R.R.; Lilenbaum, W. (2010). Species distribution and antimicrobial susceptibility of staphylococci isolated from canine otitis externa. Vet. Dermatol. 21, 292-296.

10. Prescott, J.F.; Hanna, W.J.B.; Smith, R.R.; Drost, K. (2002). Antimicrobial drug use and resistance in dogs. Can. Vet. J. 43, 107-116.

11. Rubin, J.; Walker, R.D.; Blickenstaff, K.; Bodeis-Jones, S.; Zhao, S. (2008) Antimicrobial resistance and genetic characterization of fluoroquinolone resistance of Pseudomonas aeruginosa isolated from canine infections. Vet. Microbiol. 131, 164-172.

12. Schick, A.E; Angus, J.C.; Coyner, K.S. (2007). Variability of laboratory identification and antibiotic susceptibility reporting of Pseudomonas spp. isolates from dogs with chronic otitis externa. Vet. Dermatol. 18, 120126.

13. Wildermuth, B.E.; Griffin, C.E.; Rosenkrantz, W.S.; Boord, M.J. (2007) Susceptibility of Pseudomonas isolates from the ears and skin of dogs to enrofloxacin, marbofloxacin, and ciprofloxacin. J. Am. Anim. Hosp. Assoc. 43, 337-341. 